\title{
Invariants of Surface Indicatrix in a Special Linear Transformation
}

\author{
A. Artykbaev ${ }^{1, *}$, B. M. Sultanov ${ }^{2}$ \\ ${ }^{1}$ Railway Engineering Institute, Uzbekistan \\ ${ }^{2}$ Department of Geometry and Topology, National University of Uzbekistan, Uzbekistan
}

Received June 16, 2019; Revised August 12, 2019; Accepted August 22, 2019

Copyright $\odot 2019$ by authors, all rights reserved. Authors agree that this article remains permanently open access under the terms of the Creative Commons Attribution License 4.0 International License

\begin{abstract}
The linear transformation of the plane is considered, whose matrix belongs to the Heisenberg group. The transformation matrix is neither symmetric nor orthogonal. But the determinant is one. The class of the second-order curves is studied, which is obtained from each other by the transformation under consideration. The invariant values of curves of this class are proved. In particular, the conservation of the product of semi-axes of curves in this class is proved, as well as the equality of the areas for the ellipses of the class under consideration. The obtained invariants of the second order curves are applied to curves of the second order, which is the indicatrix of the surface. Conclusion: a theorem is obtained which proves the invariance of the total curvature of a surface in a Euclidean space of the class under consideration is a transformation, which is a deformation.
\end{abstract}

Keywords Second Order Curve, Heisenberg Group, Curve Indicatrix, Surface, Gauss Curvature, Total Curvature, Principal Curvature

\section{Introduction}

The invariant is one of the main concepts in mathematics. When we consider the concept of the invariant, we understand a number or an algebraic expression associated with the figures, which keeps their values when they perform a certain transformation on that figure. We refer to the invariants of motion groups. They help to consider the important problems. In addition to these invariants, the invariants are also involved when determining parameters of a surface, a line, that's getting two parameters instead of one parameter.

We determine constants in the following linear transformation in Euclidean space:

$$
\left\{\begin{array}{l}
x^{\prime}=x+a \\
y^{\prime}=h x+y+b
\end{array} \quad-\infty \leq h<+\infty .\right.
$$

This transformation comprises parallel motion of $\vec{a}=(a, b)$ and $A=\left(\begin{array}{ll}1 & 0 \\ h & 1\end{array}\right)$ matrix transformation [1]. Here $\operatorname{Det} A=1$. Matrix $A$ is an element of Heisenberg group [8;4;3]. Let $a=b=0$ in (1). Then (1) is written as

$$
\left\{\begin{array}{l}
x^{\prime}=x \\
y^{\prime}=h x+y
\end{array}\right.
$$

Taking a line $x=x_{0}$ parallel to the $O y$ axis converts linear transformation into

$$
\left\{\begin{array}{l}
x^{\prime}=x_{0} \\
y^{\prime}=h x_{0}+y
\end{array}\right.
$$

Equality $x^{\prime}=x_{0}$ asserts that line does not change and from the equality $y^{\prime}=h x_{0}+y$ we can see that the line slides a distance of $h x_{0}$ (Fig 1 ).

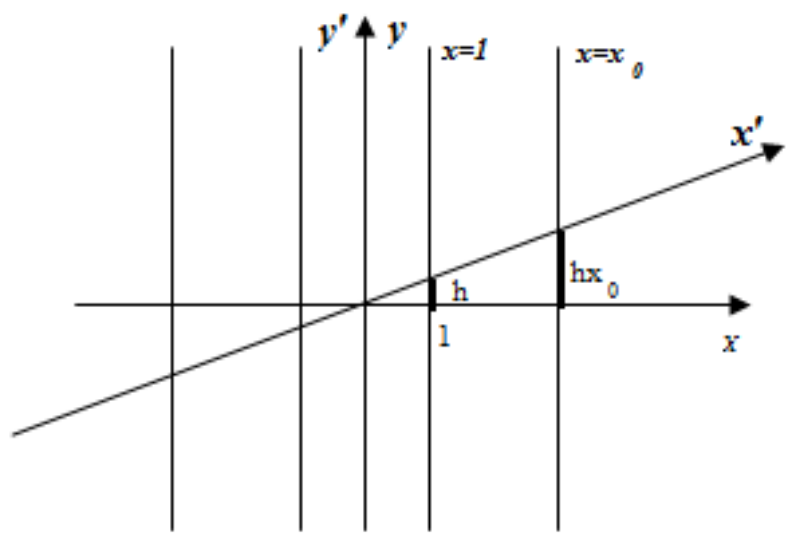

Figure 1. Linear transformation in plane

Let be given a surface $F$ in Euclidean space $R_{3}$ and 
$M \in F, \Pi$ is a tangent plane taken at $M$ node.

Our objective is to learn a curvature of the surface when a linear transformation holds for the indicatrix equation of the surface (2).

\section{Preliminaries}

Let surface $F$ be given by the vector function below:

$$
\begin{aligned}
& \stackrel{r}{r}=\stackrel{r}{r}(u, v)=x(u, v) \stackrel{i}{i}+y(u, v) \stackrel{\prime}{j}+z(u, v) \stackrel{r}{k} \\
& (u, v) \in D \text {. }
\end{aligned}
$$

We take a regular $\gamma$ line:

$$
\vec{r}=\vec{r}(s) ; x=x(s) ; y=y(s) ; z=z(s) .
$$

By denoting $k$ curvature of the line $\gamma$ given by (4), its unit normal $\vec{n}$ at the point $M$ of the surface, the angle $\theta$ between the principal normal at that point and vector $\vec{n}$, we get the following equality for the normal curvature at the point $M$ of the line lying on the surface [7;9]:

$$
k_{n}=k \cos \theta=\frac{L d u^{2}+2 M d u d v+N d v^{2}}{E d u^{2}+2 F d u d v+N d v^{2}} .
$$

Here is $k$ - curvature of the line, $\theta$ - angle between $\vec{n}$ and $\vec{v}$ normal vectors, $E, F, G ; L, M, N$ are the first and the second fundamental form coefficients respectively.

We rewrite (5) in a following way:

$$
k_{n}=\frac{1}{R}=L\left(\frac{d u}{d s}\right)^{2}+2 M\left(\frac{d u}{d s}\right)\left(\frac{d v}{d s}\right)+N\left(\frac{d v}{d s}\right)^{2} .
$$

If an intersecting plane rotates around its normal, that is if $d u: d v$ changes, then $k_{n}$ curvature of appropriate normal section changes. We take such a section $\overline{M P}$ for each normal section of tangent plane at $M$ node of surface that the following equality holds:

$$
\overline{M P}=\sqrt{|R|} \text {. }
$$

Where $R$ is a curvature radius of the section.

Geometric position of the node $P$ constituted in the tangent plane is called curvature indicatrix [10]. These yields to

$$
k_{n}=\frac{1}{(\overline{M P})^{2}} .
$$

Normal sections corresponding to the principal directions of indicatrix are called principal sections, while their curvatures are principal curvature. The product of principal curvatures at a given are called total curvatures
[5].

In addition Gauss curvature for a surface is determined and Gauss curvature of the surface is equal to the absolute value of the total curvature [2].

Let be given a second order curve $\gamma$ in the tangent plane by the following equation:

$$
a_{11} x^{2}+2 a_{12} x y+a_{22} y^{2}+2 a_{13} x+2 a_{23} y+a_{33}=0
$$

Then for the direction $\{l, m\}$ the curve

$$
\left(a_{11} l+a_{12} m\right) x+\left(a_{12} l+a_{22} m\right) y+a_{13} l+a_{22} m=0
$$

is a conjugate diameter to the direction $\{l, m\}$ [6].

Moreover, if $\left\{l^{\prime}, m^{\prime}\right\}$ and $\{l, m\}$ are directions of conjugate diameters, then the next equality holds [6]:

$$
l^{\prime}: m^{\prime}=-\left(a_{12} l+a_{22} m\right):\left(a_{11} l+a_{12} m\right) .
$$

It is known that, orthogonal and conjugate directions are named as the principal directions of a second order curve [11]. The orthogonality condition is written as

$$
l l^{\prime}+m m^{\prime}=0 .
$$

According to (8), in order the direction $\{l, m\}$ to be the principal direction; it has to be conjugate to the direction $\{-m, l\}$. Indeed, if $\{l, m\}$ is direction, $\{-m, l\}$ is also principal direction.

It follows from (8) that for the principal directions of the curve given by (7) hold the following condition [11]:

$$
a_{12} I^{2}+\operatorname{lm}\left(a_{22}-a_{11}\right)-a_{12} m^{2}=0 \text {. }
$$

\section{Main Results}

Let $F$ be a convex surface. It is known that if all points of a surface in Euclidean space are composed by elliptic points, this surface is said to be convex surface. Curvature indicatrix in tangent plane of the convex surface forms ellipses. Let curvature indicatrix in tangent plane $\Pi$ to the convex surface $F$ at the point $M$ be given by the following equation

$$
\frac{x^{2}}{a^{2}}+\frac{y^{2}}{b^{2}}=1
$$

We define invariants of ellipse given by curvature indicatrix (10) in the following linear transformation

$$
\left\{\begin{array}{l}
x^{\prime}=x \\
y^{\prime}=y-\frac{h}{a} x
\end{array}\right.
$$

Property 3.1. When the second order curve given by the equation (10) is linearly transformed by (11) all points of 
ellipse which are $x=x_{0} \neq 0,-a \leq x_{0} \leq a$ slip off the distance $\frac{h}{a} x_{0}$ parallel to $O y$ while the points $(0, b)$ and $(0,-b)$ remain unmoved.

One can prove this property by applying (11) linear transformation to (10).

Applying linear transformation (11) to (10) the equation of ellipse is written as

$$
\left(b^{2}+h^{2}\right) x^{2}-2 a h x y+a^{2} y^{2}-a^{2} b^{2}=0 .
$$

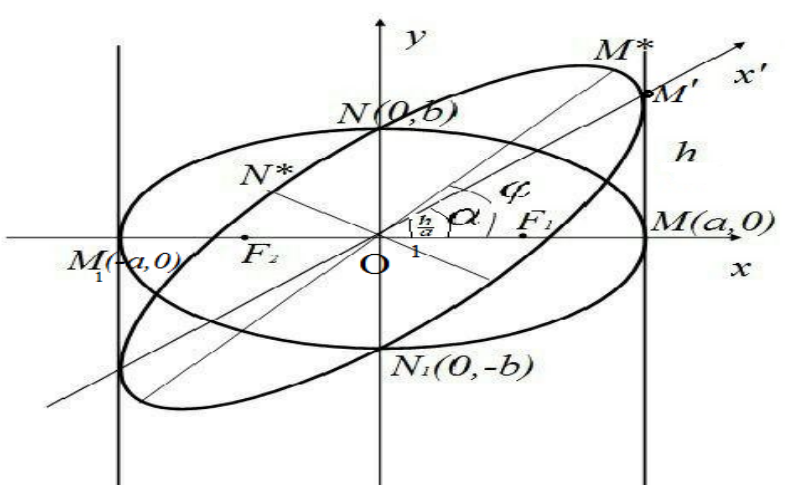

Figure 2. Curvature indicatrix is Ellipse

For the given direction $\{l, m\}$ the principal directions of ellipse (12) obtained from (9) are as follows:

$$
\frac{l}{m}=\cot \varphi_{1,2}=\frac{\left(h^{2}-c^{2}\right) \pm \sqrt{\left(h^{2}-c^{2}\right)^{2}+4 a^{2} h^{2}}}{-2 a h} \text {. }
$$

Here $\quad c^{2}=a^{2}-b^{2} \quad, \quad \alpha=<M O M^{\prime} \quad$ and $\varphi=<M O M^{*}$ (Fig 2).

Theorem 3.1. Let the surface indicatrix be given with the equation (10). Then if the linear transformation (11) is satisfied, then the equality below holds:

$$
\frac{1}{(O M)^{2}} \cdot \frac{1}{(O N)^{2}}=\frac{1}{\left(O M^{*}\right)^{2}} \cdot \frac{1}{\left(O N^{*}\right)^{2}}=\frac{1}{a^{2} b^{2}} \text {. }
$$

Proof. If principal directions of the ellipse given by the equation makes an angle $\varphi$ with the axis $O x$, equations of curves passing through principal directions take the following forms:

$$
\begin{aligned}
& O M^{*}: y_{1}=\tan \varphi_{1} \cdot x=\frac{\sqrt{\left(h^{2}-c^{2}\right)^{2}+4 a^{2} h^{2}}-\left(c^{2}-h^{2}\right)}{2 a h} x \\
& O N^{*}: y_{2}=\tan \varphi_{2} \cdot x=\frac{\sqrt{\left(h^{2}-c^{2}\right)^{2}+4 a^{2} h^{2}}+\left(c^{2}-h^{2}\right)}{-2 a h} x
\end{aligned}
$$

Now we find the point of intersection of these equations with the ellipse. Then we obtain the distance between the origin of ellipse and the intersection point:

$$
\begin{aligned}
& d_{1}^{2}=\left(O M^{*}\right)^{2}=\left|\frac{\left(\sqrt{\left(h^{2}-c^{2}\right)^{2}+4 a^{2} h^{2}}+\left(h^{2}-c^{2}\right)\right) b^{2}}{\sqrt{\left(h^{2}-c^{2}\right)^{2}+4 a^{2} h^{2}}-\left(h^{2}+c^{2}\right)}\right| \\
& d_{2}{ }^{2}=\left(O N^{*}\right)^{2}=\left|\frac{\left(\sqrt{\left(h^{2}-c^{2}\right)^{2}+4 a^{2} h^{2}}-\left(h^{2}-c^{2}\right)\right) b^{2}}{\sqrt{\left(h^{2}-c^{2}\right)^{2}+4 a^{2} h^{2}}+\left(h^{2}+c^{2}\right)}\right|
\end{aligned}
$$

and hence

$$
\frac{1}{\left(O M^{*}\right)^{2}}=\left|\frac{h^{2}+a^{2}+b^{2}-\sqrt{\left(h^{2}-c^{2}\right)^{2}+4 a^{2} h^{2}}}{2 a^{2} b^{2}}\right|
$$

$$
\frac{1}{\left(O N^{*}\right)^{2}}=\left|\frac{h^{2}+a^{2}+b^{2}+\sqrt{\left(h^{2}-c^{2}\right)^{2}+4 a^{2} h^{2}}}{2 a^{2} b^{2}}\right|
$$

Finally

$$
\begin{gathered}
\frac{1}{\left(O M^{*}\right)^{2}} \cdot \frac{1}{\left(O N^{*}\right)^{2}}=\left|\frac{h^{2}+a^{2}+b^{2}-\sqrt{\left(h^{2}-c^{2}\right)^{2}+4 a^{2} h^{2}}}{2 a^{2} b^{2}} \cdot \frac{h^{2}+a^{2}+b^{2}+\sqrt{\left(h^{2}-c^{2}\right)^{2}+4 a^{2} h^{2}}}{2 a^{2} b^{2}}\right|= \\
=\left|\frac{\left(a^{2}+b^{2}\right)^{2}-c^{4}}{4 a^{4} b^{4}}\right|=\frac{1}{a^{2} b^{2}} .
\end{gathered}
$$

So $(O M)^{2}=a^{2},(O N)^{2}=b^{2}$. Theorem proved.

Corollary 3.1. In linear transformation (11) the area of ellipse given by the equation (10) doesn't change (Fig 2).

Proof. We know from the course of analytic geometry that the area of the ellipse is equal to

$$
S=a b \pi \text {. }
$$

It yields that the area of the ellipse given by the equation (10) is equal to (13). In linear transformation, ellipse (12) shifts to an ellipse. Now we find the area of the ellipse (12). Semi - major and semi - minor axis of the ellipse (12) are 


$$
\begin{aligned}
& a^{*}=\sqrt{\left|\frac{\left(\sqrt{\left(h^{2}-c^{2}\right)^{2}+4 a^{2} h^{2}}+\left(h^{2}-c^{2}\right)\right) b^{2}}{\sqrt{\left(h^{2}-c^{2}\right)^{2}+4 a^{2} h^{2}}-\left(h^{2}+c^{2}\right)}\right|} \\
& b^{*}=\sqrt{\frac{\left(\sqrt{\left(h^{2}-c^{2}\right)^{2}+4 a^{2} h^{2}}-\left(h^{2}-c^{2}\right)\right) b^{2}}{\sqrt{\left(h^{2}-c^{2}\right)^{2}+4 a^{2} h^{2}}+\left(h^{2}+c^{2}\right)} \mid}
\end{aligned}
$$

Its area equals to

$$
\begin{gathered}
S=a^{*} b^{*} \pi=\sqrt{\left|\frac{\left(\sqrt{\left(h^{2}-c^{2}\right)^{2}+4 a^{2} h^{2}}+\left(h^{2}-c^{2}\right)\right) b^{2}}{\sqrt{\left(h^{2}-c^{2}\right)^{2}+4 a^{2} h^{2}}-\left(h^{2}+c^{2}\right)}\right|} \cdot \sqrt{\mid \frac{\left(\sqrt{\left(h^{2}-c^{2}\right)^{2}+4 a^{2} h^{2}}-\left(h^{2}-c^{2}\right)\right) b^{2}}{\sqrt{\left(h^{2}-c^{2}\right)^{2}+4 a^{2} h^{2}}+\left(h^{2}+c^{2}\right)}=} \\
=\sqrt{\left|\frac{4 a^{4} b^{4}}{\left(a^{2}+b^{2}\right)^{2}-c^{4}}\right|} \cdot \pi=\sqrt{a^{2} b^{2}} \cdot \pi=a b \pi .
\end{gathered}
$$

So the area of the ellipse (10) in linear transformation (11) does not change.

So it takes to the following theorem.

Theorem 3.2. Curvature indicatrix of a convex surface whose all points are elliptic at the point $M$ in linear transformation (2), the Gauss curvature at the point does not change.

Proof. The proof follows from the previous theorem. Let curvature indicatrix of the surface $F$ be given by the equation (10). Then when the curvature indicatrix is linearly transformed by (11), it converts to the surface $F^{*}$ whose indicatrix equation given by (12).

We obtain principal curvatures of these surfaces from the equality (6). The principal curvatures of the surface whose curvature indicatrix given by the equation (10) are

$$
F: k_{1}=\frac{1}{(O M)^{2}}=\frac{1}{a^{2}} ; k_{2}=\frac{1}{(O N)^{2}}=\frac{1}{b^{2}} .
$$

total curvature is equal to

$$
K_{1}=k_{1} k_{2}=\frac{1}{a^{2} b^{2}} .
$$

We have principal curvatures of the surface whose curvature indicatrix given by the equation (12) are found from (6) as

$$
F^{*}:\left\{\begin{array}{l}
k_{1}=\frac{1}{\left(O M^{*}\right)^{2}}=\left|\frac{h^{2}+a^{2}+b^{2}-\sqrt{\left(h^{2}-c^{2}\right)^{2}+4 a^{2} h^{2}}}{2 a^{2} b^{2}}\right| \\
k_{2}=\frac{1}{\left(O N^{*}\right)^{2}}=\left|\frac{h^{2}+a^{2}+b^{2}+\sqrt{\left(h^{2}-c^{2}\right)^{2}+4 a^{2} h^{2}}}{2 a^{2} b^{2}}\right|
\end{array}\right.
$$

total curvature equal to

$$
\begin{gathered}
K_{2}=k_{1} k_{2}=\frac{1}{\left(O M^{*}\right)^{2}} \cdot \frac{1}{\left(O N^{*}\right)^{2}}=\left|\frac{h^{2}+a^{2}+b^{2}-\sqrt{\left(h^{2}-c^{2}\right)^{2}+4 a^{2} h^{2}}}{2 a^{2} b^{2}} \cdot \frac{h^{2}+a^{2}+b^{2}+\sqrt{\left(h^{2}-c^{2}\right)^{2}+4 a^{2} h^{2}}}{2 a^{2} b^{2}}\right|= \\
=\left|\frac{\left(a^{2}+b^{2}\right)^{2}-c^{4}}{4 a^{4} b^{4}}\right|=\frac{1}{a^{2} b^{2}} .
\end{gathered}
$$

It follows that total (Gauss) curvature doesn't change:

$$
K_{1}=K_{2}
$$


We proved geometrically that total curvature of a surface doesn't change.

Now we study invariants of curvature indicatrix of saddle surfaces in linear transformation (2).

Let $F$ be a saddle surface. It is known that if all points of a surface in Euclidean space are composed by hyperbolic points, this surface is said to be saddle surface. Curvature indicatrix in tangent plane of the saddle surface forms hyperbolas. Let curvature indicatrix in tangent plane $\Pi$ to the saddle surface $F$ at the point $M$ be given by the following equation

$$
\frac{x^{2}}{a^{2}}-\frac{y^{2}}{b^{2}}= \pm 1
$$

We define invariants of hyperbola given by curvature indicatrix (14) in the linear transformation (11).

Applying linear transformation (11) to the equation of hyperbola (14) is rewritten as

$$
\left(b^{2}-h^{2}\right) x^{2}+2 a h x y-a^{2} y^{2} \mp a^{2} b^{2}=0 \text {. }
$$

For the given direction $\{l, m\}$ the principal directions of hyperbola (15) are obtained from (9) as

$$
\frac{l}{m}=\cot \varphi_{1,2}=\frac{\left(c^{2}-h^{2}\right) \pm \sqrt{\left(h^{2}-c^{2}\right)^{2}+4 a^{2} h^{2}}}{2 a h}
$$

Here $c^{2}=a^{2}+b^{2}$ (Fig 3).

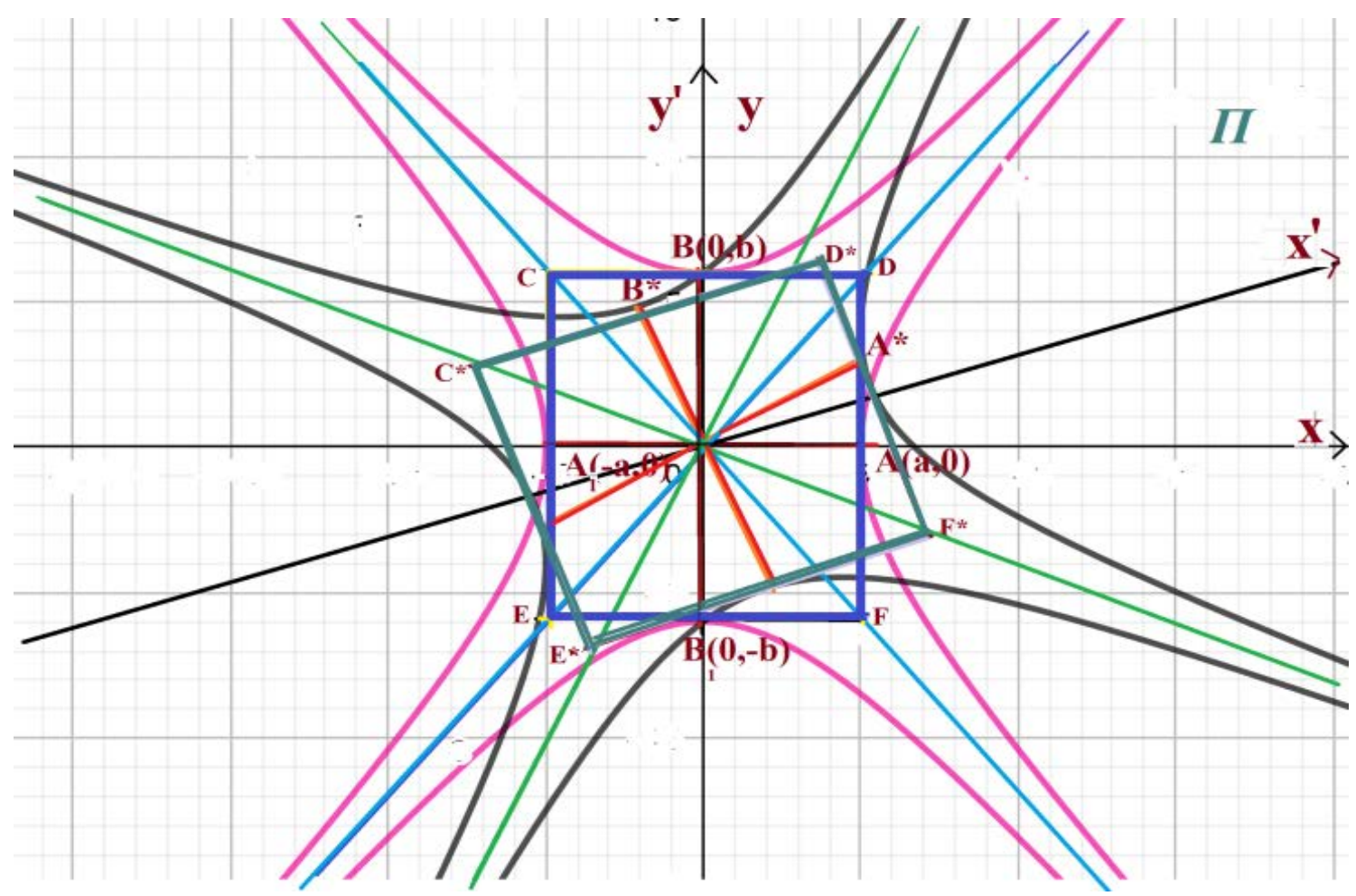

Figure 3. Curvature indicatrix is Hyperbola

Theorem 3.3. Let the surface indicatrix be given with the equation of (14). If the linear transformation (11) holds for (14), then the equality below holds:

$$
\frac{1}{(O A)^{2}} \cdot \frac{1}{(O B)^{2}}=\frac{1}{\left(O A^{*}\right)^{2}} \cdot \frac{1}{\left(O B^{*}\right)^{2}}=\frac{1}{a^{2} b^{2}}
$$

Proof. If the principal directions of a hyperbola (15) makes an angle $\varphi$ with the axis $O x$, then the equations of the lines passing through the principal directions take the next form: 


$$
\begin{aligned}
& O A^{*}: y_{1}=\tan \varphi_{1} \cdot x=\frac{\left(c^{2}-h^{2}\right)-\sqrt{\left(h^{2}-c^{2}\right)^{2}+4 a^{2} h^{2}}}{-2 a h} x \\
& O B^{*}: y_{2}=\tan \varphi_{2} \cdot x=\frac{\left(c^{2}-h^{2}\right)+\sqrt{\left(h^{2}-c^{2}\right)^{2}+4 a^{2} h^{2}}}{-2 a h} x .
\end{aligned}
$$

We seek the intersection points of these equations with the hyperbola. And then we find the distances between the origin of the hyperbola and intersection points:

$$
\begin{aligned}
& d_{1}^{2}=\left(O A^{*}\right)^{2}=\left|\frac{\left(\sqrt{\left(c^{2}-h^{2}\right)^{2}+4 a^{2} h^{2}}-\left(c^{2}-h^{2}\right)\right) b^{2}}{\left(c^{2}+h^{2}\right)-\sqrt{\left(c^{2}-h^{2}\right)+4 a^{2} h^{2}}}\right| \\
& d_{2}^{2}=\left(O B^{*}\right)^{2}=\left|\frac{\left(\sqrt{\left(h^{2}-c^{2}\right)^{2}+4 a^{2} h^{2}}+\left(c^{2}-h^{2}\right)\right)\left(-b^{2}\right)}{\left(h^{2}+c^{2}\right)+\sqrt{\left(h^{2}-c^{2}\right)+4 a^{2} h^{2}}}\right|
\end{aligned}
$$

These yields to

$$
\begin{aligned}
& \frac{1}{\left(O A^{*}\right)^{2}}=\left|\frac{b^{2}-\left(h^{2}+a^{2}\right)+\sqrt{\left(c^{2}-h^{2}\right)^{2}+4 a^{2} h^{2}}}{2 a^{2} b^{2}}\right| \\
& \frac{1}{\left(O B^{*}\right)^{2}}=\left|\frac{b^{2}-\left(h^{2}+a^{2}\right)-\sqrt{\left(c^{2}-h^{2}\right)^{2}+4 a^{2} h^{2}}}{2 a^{2} b^{2}}\right|
\end{aligned}
$$

and

$$
\begin{gathered}
\frac{1}{\left(O A^{*}\right)^{2}} \cdot \frac{1}{\left(O B^{*}\right)^{2}}=\left|\frac{b^{2}-\left(h^{2}+a^{2}\right)+\sqrt{\left(c^{2}-h^{2}\right)+4 a^{2} h^{2}}}{2 a^{2} b^{2}} \cdot \frac{b^{2}-\left(h^{2}+a^{2}\right)-\sqrt{\left(c^{2}-h^{2}\right)+4 a^{2} h^{2}}}{2 a^{2} b^{2}}\right|= \\
=\left|\frac{\left(a^{2}-b^{2}\right)^{2}-c^{4}}{4 a^{4} b^{4}}\right|=\frac{1}{a^{2} b^{2}} .
\end{gathered}
$$

So, $(O M)^{2}=a^{2},(O N)^{2}=b^{2}$. Theorem proved.

Corollary 3.2. When we consider hyperbola (14) in linear transformation (11), areas of the rectangles $C D E F$ and $C^{\prime} D^{\prime} E^{\prime} F^{\prime}$ are equal (Fig 3).

Proof. It is known that, the area of a rectangle whose sides are $a, b$ is computed by the following formula

$$
S=a b \text {. }
$$

In figure 3, the sides of the rectangle $C D E F$ are $C D=E F=2 a, C E=D F=2 b$. The area of the rectangle $C D E F$ is

$$
S=4 a b .
$$

After linear transformation hyperbola (15) rectangle $C D E F$ shifts to the rectangle $C^{\prime} D^{\prime} E^{\prime} F^{\prime}$. The sides of the rectangle $C^{\prime} D^{\prime} E^{\prime} F^{\prime}$ are determined as follows:

$$
C^{\prime} D^{\prime}=E^{\prime} F^{\prime}=2 \cdot \sqrt{\frac{\left(\sqrt{\left(c^{2}-h^{2}\right)^{2}+4 a^{2} h^{2}}-\left(c^{2}-h^{2}\right)\right) b^{2}}{\left(c^{2}+h^{2}\right)-\sqrt{\left(c^{2}-h^{2}\right)+4 a^{2} h^{2}}}}
$$




$$
C^{\prime} E^{\prime}=D^{\prime} F^{\prime}=2 \cdot \sqrt{\frac{\left(\sqrt{\left(h^{2}-c^{2}\right)^{2}+4 a^{2} h^{2}}+\left(c^{2}-h^{2}\right)\right)\left(-b^{2}\right)}{\left(h^{2}+c^{2}\right)+\sqrt{\left(h^{2}-c^{2}\right)+4 a^{2} h^{2}}}}
$$

Its area equals to

$$
\begin{gathered}
S=2 \cdot \sqrt{\left|\frac{\left(\sqrt{\left(c^{2}-h^{2}\right)^{2}+4 a^{2} h^{2}}-\left(c^{2}-h^{2}\right)\right) b^{2}}{\left(c^{2}+h^{2}\right)-\sqrt{\left(c^{2}-h^{2}\right)+4 a^{2} h^{2}}}\right| \cdot 2 \cdot \sqrt{\left|\frac{\left(\sqrt{\left(h^{2}-c^{2}\right)^{2}+4 a^{2} h^{2}}+\left(c^{2}-h^{2}\right)\right)\left(-b^{2}\right)}{\left(h^{2}+c^{2}\right)+\sqrt{\left(h^{2}-c^{2}\right)+4 a^{2} h^{2}}}\right|}=} \\
=4 \cdot \sqrt{\left|\frac{4 a^{4} b^{4}}{\left(a^{2}-b^{2}\right)^{2}-c^{4}}\right|}=4 \cdot \sqrt{a^{2} b^{2}}=4 a b
\end{gathered}
$$

So, the areas of $C D E F$ and $C^{\prime} D^{\prime} E^{\prime} F^{\prime}$ of the rectangles are equal.

Theorem 3.4. The Gauss curvature at the point $M$ does not change in linear transformation (2) of curvature indicatrix at the point $M$ of a saddle surface whose all points are hyperbolic points.

Proof. The proof follows from the previous theorem. Let the curvature indicatrix of the surface $F$ be given by (14). Then by carrying out linear transformation (11) to its curvature indicatrix it is converted to the surface $F^{*}$ with the curvature indicatrix equation (15).

Remark 3.1. In saddle surfaces normal curvatures of the sections with downward concavity are less than zero while with upward concavity they are greater than zero. In this case $K=k_{1} \cdot k_{2}<0$, that is $k_{1}$ are $k_{2}$ different signs, for example:

$$
k_{1}>0, k_{2}<0
$$

We get principal curvatures of these surfaces from (6). The principal curvatures of the surface with the curvature indicatrix equation (14) are obtained from (6):

$$
F: k_{1}=\frac{1}{(O A)^{2}}=\frac{1}{a^{2}} ; k_{2}=-\frac{1}{(O B)^{2}}=-\frac{1}{b^{2}} .
$$

We assume that concave of the first principal section is directed to $\vec{n}$ and of the second to $-\vec{n}$. Normal curvature is positive for some directions and negative for some.

Total curvature has the form

$$
K_{1}=k_{1} k_{2}=-\frac{1}{a^{2} b^{2}} .
$$

We get principal curvatures of the surface formed in linear transformation with the curvature indicatrix equation (15) from (6)

$$
F^{*}:\left\{\begin{array}{l}
k_{1}=\frac{1}{\left(O A^{*}\right)^{2}}=\left|\frac{b^{2}-\left(h^{2}+a^{2}\right)+\sqrt{\left(c^{2}-h^{2}\right)^{2}+4 a^{2} h^{2}}}{2 a^{2} b^{2}}\right| \\
k_{2}=-\frac{1}{\left(O B^{*}\right)^{2}}=-\left|\frac{b^{2}-\left(h^{2}+a^{2}\right)-\sqrt{\left(c^{2}-h^{2}\right)^{2}+4 a^{2} h^{2}}}{2 a^{2} b^{2}}\right|
\end{array}\right.
$$

total curvature equal to

$$
\begin{gathered}
K_{2}=k_{1} k_{2}=\frac{1}{\left(O A^{*}\right)^{2}} \cdot \frac{1}{\left(O B^{*}\right)^{2}}=-\left|\frac{b^{2}-\left(h^{2}+a^{2}\right)+\sqrt{\left(c^{2}-h^{2}\right)^{2}+4 a^{2} h^{2}}}{2 a^{2} b^{2}} \cdot \frac{b^{2}-\left(h^{2}+a^{2}\right)-\sqrt{\left(c^{2}-h^{2}\right)^{2}+4 a^{2} h^{2}}}{2 a^{2} b^{2}}\right|= \\
=-\left|\frac{\left(a^{2}-b^{2}\right)^{2}-c^{4}}{4 a^{4} b^{4}}\right|=-\frac{1}{a^{2} b^{2}} .
\end{gathered}
$$

It follows from above expression that (total) Gauss curvature doesn’t change: 


$$
K_{1}=K_{2} \text {. }
$$

We proved geometrically that total curvature in linear transformation of the surface at a saddle point doesn't move.

As we experienced linear transformation (1) the lines parallel to $O y$ transfer the lines again parallel to $O y$. Asymptotes of the hyperbola (14) are not parallel to $O y$. It is not possible to shift the hyperbola given by (14) to the hyperbola whose asymptotes are the $O y$ axis in linear transformation (1). Therefore, we study separately the hyperbola whose asymptotes are located on the $O y$ axis.

Let the given $L$ be a hyperbola with coordinate axes of the asymptotes of the curvature index of the plane of $\Pi$, transferred to the point $M$ of the surface. We make a linear transformation (17) to the surface given by the equation

$$
\begin{gathered}
y= \pm \frac{k}{x} \\
\left\{\begin{array}{l}
x^{\prime}=x \\
y^{\prime}=y-\frac{h}{k} x .
\end{array}\right.
\end{gathered}
$$

We determine the constants in this transformation. Hyperbola equation (16) after the linear transformation (17) takes the form (Fig 4):

$$
k x y-h x^{2} \mp k^{2}=0 .
$$

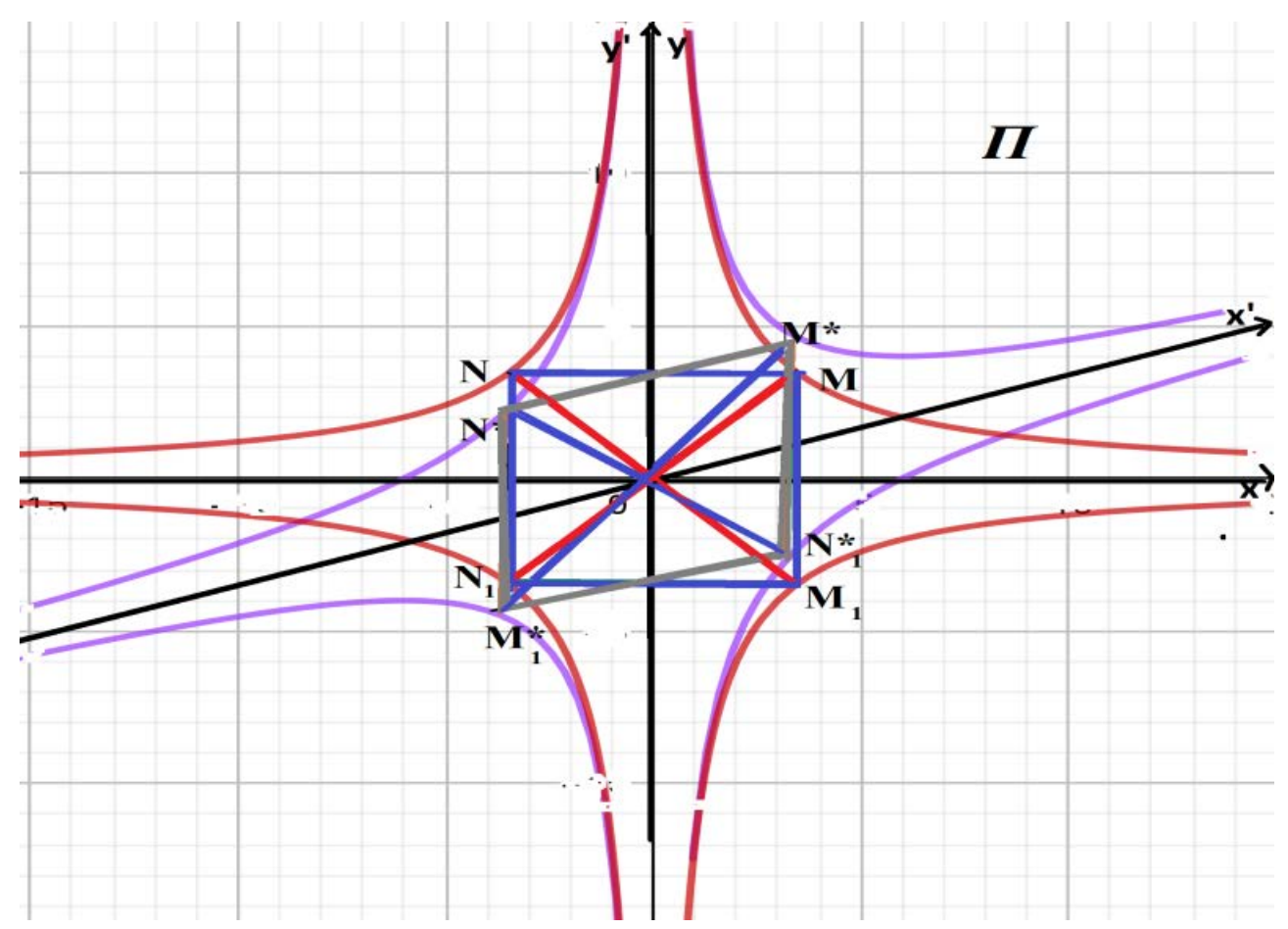

Figure 4. Curvature indicatrix is special Hyperbola.

For the given direction $\{l, m\}$ the principal directions of hyperbola (18) are obtained from (9) has the form:

$$
\frac{l}{m}=\cot \varphi_{1,2}=\frac{-h \pm \sqrt{h^{2}+k^{2}}}{k}
$$

Theorem 3.5. Let surface indicatrix be given by (16). If we accomplish linear transformation (17), then it holds the following equality: 


$$
\frac{1}{(O M)^{2}} \cdot \frac{1}{(O N)^{2}}=\frac{1}{\left(O M^{*}\right)^{2}} \cdot \frac{1}{\left(O N^{*}\right)^{2}}=\frac{1}{4 k^{2}}
$$

Proof. If the principal directions of hyperbola (18) makes an angle $\varphi$ with the axis $O x$, then the lines passing through the principal directions has the forms

$$
\begin{aligned}
& O M^{*}: y_{1}=\tan \varphi_{1} \cdot x=\frac{h-\sqrt{h^{2}+k^{2}}}{k} x \\
& O N^{*}: y_{2}=\tan \varphi_{2} \cdot x=\frac{h+\sqrt{h^{2}+k^{2}}}{k} x
\end{aligned}
$$

We find the points of intersection of these equations with hyperbola. Then we compare the distance between the origin of hyperbola and the points of intersection:

$$
\begin{aligned}
& d_{1}^{2}=\left(O M^{*}\right)^{2}=\left|-2\left(\sqrt{h^{2}+k^{2}}-h\right)\right| \\
& d_{2}{ }^{2}=\left(O N^{*}\right)^{2}=\left|2\left(\sqrt{h^{2}+k^{2}}+h\right)\right|
\end{aligned}
$$

From this, we have

$$
\begin{aligned}
& \frac{1}{\left(O M^{*}\right)^{2}}=\left|\frac{1}{-2\left(\sqrt{h^{2}+k^{2}}-h\right)}\right| \\
& \frac{1}{\left(O N^{*}\right)^{2}}=\left|\frac{1}{2\left(\sqrt{h^{2}+k^{2}}+h\right)}\right|
\end{aligned}
$$

Hence

$$
\frac{1}{\left(O M^{*}\right)^{2}} \cdot \frac{1}{\left(O N^{*}\right)^{2}}=\left|\frac{1}{-2\left(\sqrt{h^{2}+k^{2}}-h\right)} \cdot \frac{1}{2\left(\sqrt{h^{2}+k^{2}}+h\right)}\right|=\frac{1}{4 k^{2}}
$$

So $\left(O M^{2}\right)=2 k,\left(O N^{2}\right)=2 k$. Theorem is proved.

We arrive at the following proposition.

Proposition 3.3. The areas of $M N M_{1} N_{1}$ square and $M^{*} N^{*} M_{1}^{*} N_{1}^{*}$ rhombus are equal (Fig 4).

Proof. In transformation (17), $M N M_{1} N_{1}$ square shift to $M^{*} N^{*} M_{1}^{*} N_{1}^{*}$ rhombus. The side of square whose vertices are $M(\sqrt{k}, \sqrt{k}), M^{*}(\sqrt{k},-\sqrt{k}), N(-\sqrt{k}, \sqrt{k}), N(-\sqrt{k},-\sqrt{k})$ equals $2 \sqrt{k}$ and the area is $S=4 k$. Now we calculate the area of $M^{*} N^{*} M_{1}^{*} N_{1}^{*}$ rhombus. First we find its diagonal as follows:

$$
\begin{gathered}
d_{1}=\overline{M M}^{*}=2 \overline{O M^{*}}=2 \sqrt{\left|-2\left(\sqrt{h^{2}+k^{2}}-h\right)\right|} \\
d_{2}=\overline{N N}^{*}=2 \overline{O N^{*}}=2 \sqrt{\left|2\left(\sqrt{h^{2}+k^{2}}+h\right)\right|} .
\end{gathered}
$$

Then the area of the rhombus is

$$
S=\frac{1}{2} d_{1} d_{2}=\frac{1}{2} \cdot 2 \sqrt{\left|-2\left(\sqrt{h^{2}+k^{2}}-h\right)\right|} \cdot 2 \sqrt{\left|2\left(\sqrt{h^{2}+k^{2}}+h\right)\right|}=\frac{1}{2} \cdot 4 \sqrt{4 k^{2}}=4 k .
$$

Therefore the areas of $M N M_{1} N_{1}$ square and $M^{*} N^{*} M_{1}^{*} N_{1}^{*}$ rhombus are equal.

Theorem 3.6. Gauss curvature doesn't change at the point $M$ when we have linear transformation (2) of the surface 
with the curvature indicatrix (16).

Proof. The proof follows from the above theorem. Let curvature indicatrix of the surface $F$ be given by (16). Then when curvature indicatrix is linearly transformed by (17) it is converted to the surface $F^{*}$ with curvature indicatrix (18). We find the principal curvatures of these surfaces from (6). According to the remark 3.8, the principal curvatures of the surface with curvature indicatrix (16) are obtained (6)

$$
F: \quad k_{1}=\frac{1}{(O M)^{2}}=\frac{1}{2 k} ; k_{2}=-\frac{1}{(O N)^{2}}=-\frac{1}{2 k}
$$

Total curvature has the form

$$
K_{1}=k_{1} k_{2}=-\frac{1}{4 k^{2}} .
$$

We obtain the principal curvatures of the surface formed in linear transformation with the curvature indicatrix equation (18) from (6)

$$
F^{*}: \quad k_{1}=\frac{1}{\left(O M^{*}\right)^{2}}=\left|\frac{1}{-2\left(\sqrt{h^{2}+k^{2}}-h\right)}\right| ; k_{2}=\frac{1}{\left(O N^{*}\right)^{2}}=-\left|\frac{1}{2\left(\sqrt{h^{2}+k^{2}}+h\right)}\right|
$$

Total curvature can be written as

$$
K_{2}=k_{1} k_{2}=-\frac{1}{\left(O M^{*}\right)^{2}} \cdot \frac{1}{\left(O N^{*}\right)^{2}}=-\left|\frac{1}{-2\left(\sqrt{h^{2}+k^{2}}-h\right)} \cdot \frac{1}{2\left(\sqrt{h^{2}+k^{2}}+h\right)}\right|=-\frac{1}{4 k^{2}}
$$

This yields that total (Gauss) curvature does not change:

$$
K_{1}=K_{2}
$$

We proved geometrically that total curvature of the surface with the curvature indicatrix equation (16) in linear transformation doesn't change.

\section{Conclusions}

Obtained results show that, Gauss curvature of the surface doesn't change when special linear transformation in curvilinear coordinates is done. Linear transformation in curvilinear coordinates defines deformation bounded by the surface in Euclidean space. So, in this deformation Gauss curvature of the surface remains unchanged.

\section{REFERENCES}

[1] Artikbaev A., Sokolov D.D. Geometriya v selom v ploskom prostranstve vremeni // Tashkent, Fan, 1991. 180 p.

[2] Bakelman I.Ya., Verner A.L., Kontor B.E. Vvedenie v differensialnuyu geometriyu, v selom”, M.: Nauka, 1973, $440 \mathrm{p}$.

[3] Berdinskiy D.A. O minimalnix poverxnostyax v gruppe Geyzenberga // Vestnik KGU. № 3-1 (47) , 2011, p. 34-38.

[4] Masalsev L.A. Minimalniye poverxnosti $v$ gruppe Geyzenberga // Vestnik XNU, seriya, Matematika, prikladnaya matematika i mexanika" № 602, 2003, p. 35-45.

[5] Manfredo P. Do Carmo. Differential geometry of Curves and surface // Dover publications, Inc. Mineola, New York, 2016, p. 529.

[6] Postnikov.M.M. Analiticheskaya geometriya. Moscow, Nauka, 1973, p. 740.

[7] Poznyak E.G., Shikin E.V. Differensial geometriya // M.: Izd-vo MGU, 1990, p. 384.

[8] P.Tomter, Constant mean curvature surface in the Heisenberg group, Proe. of Symp. pure math. v54(1993), part 1, p.485-495.

[9] Toponogov V.A, Rovenski.V.Y. Differential Geometry of Curves and Surfaces. BirkhaËuser Boston, 2006, p. 214.

[10] Tolctopyatov V.P. Geometriya. Kurs leksiya // Yekaterinburg. UGPU, 2012, p. 84

Veselov A.P., Troiskiy E.V. Leksii po analiticheskoy geometrii. Moscow, Izdatelctvo MSNMO, 2017, p. 151 